\title{
Front Matter: Volume 11315
}

, "Front Matter: Volume 11315," Proc. SPIE 11315, Medical Imaging 2020: Image-Guided Procedures, Robotic Interventions, and Modeling, 1131501 (12 May 2020); doi: 10.1117/12.2570843

SPIE. Event: SPIE Medical Imaging, 2020, Houston, Texas, United States 


\title{
PROGRESS IN BIOMEDICAL OPTICS AND IMAGING
}

\section{Medical Imaging 2020}

\section{Image-Guided Procedures, Robotic Interventions, and Modeling}

\author{
Baowei Fei \\ Cristian A. Linte \\ Editors
}

16-19 February 2020

Houston, Texas, United States

Sponsored by

SPIE

Cooperating Organizations

AAPM-American Association of Physicists in Medicine (United States)

MIPS-Medical Image Perception Society (United States)

SIIM-Society for Imaging Informatics in Medicine (United States)

IFCARS - International Foundation for Computer Assisted Radiology and Surgery (Germany)

WMIS-World Molecular Imaging Society

Published by

SPIE

Volume 11315 
The papers in this volume were part of the technical conference cited on the cover and title page. Papers were selected and subject to review by the editors and conference program committee. Some conference presentations may not be available for publication. Additional papers and presentation recordings may be available online in the SPIE Digital Library at SPIEDigita lLibrary.org.

The papers reflect the work and thoughts of the authors and are published herein as submitted. The publisher is not responsible for the validity of the information or for any outcomes resulting from reliance thereon.

Please use the following format to cite material from these proceedings:

Author(s), "Title of Paper," in Medical Imaging 2020: Image-Guided Procedures, Robotic Interventions, and Modeling, edited by Baowei Fei, Cristian A. Linte, Proceedings of SPIE Vol. 11315 (SPIE, Belling ham, WA, 2020) Seven-digit Artic le CID Number.

ISSN: 1605-7422

ISSN: 2410-9045 (electronic)

ISBN: 9781510633971

ISBN: 9781510633988 (electronic)

Published by

SPIE

P.O. Box 10, Belling ham, Washington 98227-0010 USA

Telephone +1 3606763290 (Pa cific Time)· Fax +1 3606471445

SPIE.org

Copyright $@ 2020$, Society of Photo-O ptical Instrumentation Engineers.

Copying of material in this book for intemal or personal use, or for the intemal or personal use of specific clients, beyond the fair use provisions granted by the U.S. Copyright Law is a uthorized by SPIE subject to payment of copying fees. The Transactional Reporting Service base fee for this volume is $\$ 21.00$ per article (or portion thereof), which should be paid directly to the Copyright Clearance Center (CCC), 222 Rosewood Drive, Danvers, MA 01923. Payment may also be made electronically through CCC Online at copyright.com. Other copying for republication, resale, advertising or promotion, or any form of systematic or multiple reproduction of a ny material in this book is prohibited except with permission in writing from the publisher. The CCC fee code is 1605$7422 / 20 / \$ 21.00$.

Printed in the United States of America by Curran Associates, Inc., und er license from SPIE.

Publication of record for individual papers is online in the SPIE Digital Library.

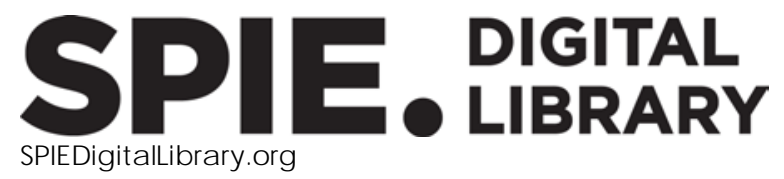

Paper Numbering: Proceedings of SPIE follow an e-First publication model. A unique citation identifier (CID) number is assigned to each article at the time of publication. Utilization of CIDs allows articles to be fully citable as soon as they are published online, and connects the same identifier to all online and print versions of the publication. SPIE uses a seven-digit CID article numbering system structured as follows:

- The first five digits correspond to the SPIE volume number.

- The last two digits indicate publication order within the volume using a Base 36 numbering system employing both numerals and letters. These two-number sets start with $00,01,02,03,04$, 05, 06, 07, 08, 09, 0A, 0B ... 0Z, followed by 10-1Z, 20-2Z, etc. The CID Number appears on each page of the manuscript. 


\title{
Contents
}

\author{
xi Authors \\ xvii Conference Committee \\ xxi Awards
}

1131502 Miniature C-am simulator using wireless accelerometer based tracking [11315-1]

1131503 Pivot calibration concept for sensor attached mobile c-ams [11315-2]

1131504 3D catheterguidance including shape sensing for endovasc ular navigation [11315-3]

1131505 Feasibility of 3D motion-compensated needle guidance for TIPS procedures [11315-4]

1131506 Towards electromagnetic tracking of J-tip guidewire: precision assessment of sensors during bending tests [11315-5]

\section{SESSION 2 AI METHODS FOR IMAGE-GUIDED THERAPY}

$1131507 \quad$ Validation of a metal artifact reduction method based on 3D conditional GANs for CT images of the ear[11315-6]

1131508 Ultrasound image simulation with generative adversarial network [11315-7]

1131509 Image registration with deep probabilistic classifiers: application in radiation therapy [11315-8]

$113150 \mathrm{~A}$ Automatic labeling of respiratory phases and detection of abnomal respiratory signals in free-breathing thoracic dynamic MR image acquisitions based on deep leaming [11315-9]

11315 OB Image-based defomable motion compensation in cone-beam CT: translation to clinical studies in interventional body radiology [11315-10]

11315 OC Stabilized ultrasound imaging of a moving object using 2D B-mode images and convolutional neural network [11315-11] 
11315 OD Infra red image-guidance for intraoperative assessment of limb length discrepancy during total hip a throplasty procedures [11315-12]

11315 OE Three-dimensional ultasound formonitoring knee inflammation and cartilage damage in osteoarthritis and meumatoid arthritis [11315-13]

11315 OF Multi-body registration for fracture reduction in orthopaedic trauma surgery (Robert F. Wagner Best Student PaperAwa id) [11315-14]

11315 OG Calibration and registration of a freehand video-guided surgical drill for orthopaedic tra uma [11315-15]

$11315 \mathrm{OH} \quad$ MR-compatible needle guidance toolkit to streamline arthrography procedures: phantom accuracy study [11315-16] 11319

11315 이 Efficient target tracking for 3D ultrasound-guided needle steering [11315-17]

11315 0J Automatic brain structure-guided registration of pre and intra-operative 3D ultrasound for neurosurgery [11315-18]

11315 OK Automatic needle localization in intraoperative 3D transvaginal ultasound images for highdose-rate interstitial gynecologic brachytherapy [11315-19]

SESSION 5 IMAGE-GUIDED NEUROSURGICAL INTERVENTIONS

$11315 \mathrm{OL} \quad$ Comparison of head pose tacking methods for mixed-reality neuronavigation for transc ranial magnetic stimulation [11315-20]

11315 OM Localisation of the subthalamic nucleus in MRI via convolutional neural networks for deep brain stimulation planning [11315-21]

$1131500 \quad$ A guidance system for electrode placement in epilepsy cases [11315-23]

11315 OP Brain deformation compensation for deep brain lead placement surgery: a comparison of simulations driven by surface vs deep brain sparse data [11315-24]

\section{SESSION 7 AI-BASED METHODS FOR TISSUE C LASSIRCATION: DIAGNOSIS AND THERAPY APPUCATIONS}

11315 0Q Classification of tumor signatures from electrosurgical vapors using mass spectrometry and machine leaming: a feasibility study [11315-25]

iv 
11315 OR Towards democratizing Al in MR-based prostate cancer diagnosis: 3.0 to 1.5 Tesla [11315-26]

11315 OS Automatic segmentation of brain tumor in intraoperative ultrasound images using 3D U-Net [11315-27]

\section{SESSION 8 KEYNOTE PRESENTATION}

11315 OT Healthcare in need of innovation: exponential technology and biomedical entrepreneurship as solution providers (Keynote Paper) [11315-28]

\section{SESSION 9 AUGMENTIE REAUTY FOR IMAGE-GUIDED THERAPY}

11315 oU Augmented reality visualization of hyperspectal imaging classifications for image-guided bra in tumor phantom resection [11315-29]

11315 OV Accuracy study of Smartglasses/ Sma itphone AR systems for perc uta neous needle interventions [11315-30]

11315 OW Augmented reality-assisted biopsy of soft tissue lesions [11315-31]

11315 0X Towards augmented reality-based suturing in monocular laparoscopic taining [11315-32]

SESSION 10 NOVEIMAG ING TECHNOLOGIES FOR INTIERENTIONALGUIDANCE

11315 oY Patient-specific deep deformation models (PSDDM) to registerplanning and intenentional ultrasound volumes in image fusion-guided intenventions [11315-33]

$113150 Z$ Image guided mitral valve replacement registration of 3D ultrasound and 2D x-ray images [11315-34]

1131510 Multi-view 3D echocardiography volume compounding for mitral valve procedure planning [11315-35]

1131511 Assessment of proton beam ablation in myocardial infarct tissue using delayed contrast enhanced magnetic resonance imaging (Updated version 28 April 2020) [11315-36]

1131512 Transformation optimization and image blending for 3D liver ultrasound series stitching [11315-37]

\section{SESSION 11 VIDEO AND OPTICALMETHODS FOR IMAG ING}

1131513 Automatic A-line coronary plaque classification using combined deep leaming and textural features in intravasc ular OCTimages [11315-38] 
1131514 Motion induced segmentation of stone fragments in ureteroscopy video [11315-39]

1131515 Evaluation of reat-time guidewire navigation using virtual endoscopic 4D fluoroscopy [11315-40]

1131516 Towards portable image guidance and automatic patient registation using an RGB-D camera and video projector [11315-41]

1131517 Open-source platform forautomated collection of training data to support video-based feedback in surgical simulators [11315-42]

1131518 Improved visual SIAM for bronchoscope tracking and registation with pre-operative CT images [11315-43]

\section{SESSION 12 ROBOT-ASSISTED IMAGE-GUIDED THERAPY}

1131519 Robotic tissue scanning with biophotonic probe [11315-44]

113151 IA Image-guided robotic k-wire placement for orthopaedic tauma surgery [11315-45]

11315 1B A mechatronic guidance system forpositron emission mammography and ultrasound-guided breast biopsy [11315-46]

11315 1C Fiducial-free 2D/3D registration of the proximal femur for robot-assisted femoroplasty [11315-47]

11315 1D Feasibility of robot-assisted ultrasound imaging with force feedback forassessment of thyroid diseases [11315-48]

\section{SESSION 13 MODEUNG APPUCATIONS FOR IMAGE-GUIDED THERAPEUTICS}

$113151 \mathrm{E} \quad$ Estimating tongue defomation during laryngoscopy using hybrid \#M-multibody model and intraoperative tacking: a cadaver pilot study [11315-49]

11315 IF The image-to-physical liver registation sparse data challenge: characterizing inverse biomechanical model resolution [11315-50]

$113151 G$ Image data-driven themal dose prediction formicrowave ablation therapy [11315-51]

$11315 \mathrm{1H} \quad$ Modeling the surgical exposure of anatomy in robot-assisted laparoscopic partial nephrectomy [11315-52] 
$1131511 \quad$ CNN-based hierarchical coarse-to-fine segmentation of pelvic CTimages forprostate cancer radiotherapy [11315-53]

11315 1] CondenseUNet a memory-efficient condensely-connected architecture forbi-ventric ular blood pool and myocardium segmentation [11315-54]

$113151 \mathrm{~K} \quad$ How well do U-Net-based segmentation tained on adult cardiac magnetic resonance imaging data generalize to rare congenital heart diseases for surgical planning? [11315-55]

$113151 \mathrm{~L} \quad$ Textual fiducial detection in breast consening surgery fora near-real time image guidance system [11315-56]

$113151 \mathrm{M} \quad$ A deep leaming approach for surgical instruments detection in Orthopaedic surgery using transfer leaming [11315-57]

SESSION 15

JOURNAL OF MEDICAL IMAGING SPECIALSECTION ON INTIRVENTIONALAND SURGICALDATA SCIENCE

$1131510 \quad$ SpineC loud: image analytics for predictive modeling of spine surgeny outcomes [11315-108]

$113151 \mathrm{~A}$ A combined radiomics and cyst fiuid inflammatory markers model to predict preoperative risk in pancreatic cystic lesions [11315-110]

11315 IR Preoperative angular insertion depth prediction in case of lateral wall cochlear implant electrode arrays [11315-111]

11315 IS Integrative radiomic analysis forpre-surgical prognostic stratification of glioblastoma patients: from advanced to basic MR protocols [11315-112]

\section{POSTER SESSION}

$113151 T \quad$ Multi-destination procedure planning for comprehensive lymph node staging bronchoscopy [11315-58]

$113151 \mathrm{U} \quad$ Virtual radia/probe endobronchial ultrasound for image-guided bronchoscopy [11315-59]

11315 IV Alignment of cortical vessels viewed through the surgical mic roscope with preoperative imaging to compensate for brain shift [11315-60]

11315 IW Rigid and deformable comections in real-time using deep leaming for prostate fusion biopsy [11315-61]

$113151 \mathrm{X} \quad$ Automated classification of brain tissue: comparison between hyperspectral imaging and diffuse refiectance spectrosc opy [11315-62] 
$113151 Y$ Automatic fiducial marker detection and localization in CTimages: a combined approach [11315-63]

$113151 Z$ Emor analysis for a navigation system using 3D abdominal ultrasound [11315-64]

1131520 Fexible piezoelectric sensor for real-time image-guided colonoscopies: a solution to endoscopic looping challenges in clinic [11315-65]

1131521 Feasibility study of catheter segmentation in 3D Frustum ultrasounds by DCNN [11315-66]

1131522 Blood flow anomaly detection via generative adversanial networks: a preliminary study [11315-67]

$1131523 \quad$ Exploiting confident information for weakly supenised prostate segmentation based on image-level labels [11315-68]

1131524 Workflow for creation and evaluation of virtual nephrolithotomy training models [11315-69]

1131525 Value based decision support to prionitize development of innovative technologies for image-guided vascular surgery in the hybrid operating theater [11315-70]

1131526 Open source software platform for interstitial ablation treatment planning [11315-71]

1131527 Automatic segmentation of spinal ultrasound landmarks with U-net using multiple consec utive images for input [11315-72]

1131528 Applications of VR medical image visualization to chordal length measurements forcardiac procedures [11315-73]

1131529 Stereovision-updated image guidance in multi-level open spine surgery: short vs. long exposure [11315-74]

$113152 \mathrm{~A}$ Assessment of skill translation of intrathecal needle insertion using realtime needle tracking with an augmented reality display [11315-75]

11315 2B Multi-slot extended view imaging on the O-Am: image qua lity and application to intra operative assessment of spinal morphology [11315-76]

$113152 \mathrm{C}$ Computer vision-guided bronchoscopic navigation using dual CNN-generated depth images and ICP registration [11315-77]

$113152 \mathrm{E} \quad$ Patient-specific, dynamic models of hypoplastic left heart syndrome tric uspid valves for simulation and planning [11315-79]

$113152 \mathrm{~F} \quad$ A Windows GUl application for real-time image guidance during motion-managed proton beam therapy [11315-80]

$113152 \mathrm{G} \quad$ Automated segmentation of computed tomography colonography images using a 3D U-Net [11315-81] 
$113152 \mathrm{H} \quad$ Spherical harmonics formodeling shape tansformations of breasts following breast surgeny [11315-82]

$1131521 \quad$ Deep leaming-based automatic prostate segmentation in 3D transrectal ultrasound images from multiple acquisition geometries and systems [11315-83]

$113152 \mathrm{~K} \quad$ Assessment of therapy applicatortargeting with a mechanically assisted 3D ultrasound system for minimally invasive focal livertumor therapy [11315-85]

$113152 \mathrm{~L} \quad$ Video-based automatic and objective endoscopic sinus surgeny skill assessment [11315-86]

11315 2M Multi-slot intraoperative imaging and 3D-2D registration for evaluation of long surgical constructs in spine surgery [11315-88]

1131520 Development of ultrasonography assistance robot for prenatal care [11315-90]

$113152 \mathrm{P}$ Data-driven detection and registration of spine surgery instrumentation in intraoperative images (Cum Laude Poster Award) [11315-91]

$113152 \mathrm{Q}$ Force and torque feedback in endoscopic vessel harvesting [11315-92]

$113152 \mathrm{R}$ Multi-step segmentation forprostate MR image based on reinforcement leaming [11315-93]

$113152 \mathrm{~S}$ Image-based extraction of breathing signal from cone-beam CT projections [11315-94]

$113152 T$ A standardized method for accuracy study of MRI-compatible robots case study: a body-mounted robot [11315-95]

$113152 \mathrm{U}$ Preoperative prediction of insertion depth of lateral wall cochlear implant electrode amays [11315-96]

$113152 \mathrm{~V}$ Cochlear implant electrode sequence optimization using patient specific neural stimulation models [11315-97]

11315 2W Renal biopsy underaugmented reality guidance (Image-Guided Procedures, Robotic Interventions, and Modeling Student PaperAward) [11315-98]

11315 2X Development of a novel tumor phantom model for head and neck squamous cell carcinoma and its applications [11315-99]

$113152 Y \quad$ Automated segmentation of cardiac chambers from cine cardiac MR using an adversarial network arc hitecture [11315-100]

$113152 Z$ Optical imaging of dental plaque pH[11315-101]

1131530 Texture kinetic features from pre-treatment DCE MRI forpredicting pathologic tumorstage regression after neoadjuvant chemoradiation in rectal cancers [11315-102]

1131531 A comprehensive workflow and fiamework for immersive virtual endoscopy of dissected aortae from CTA data [11315-104] 
1131532 A personalized approach formic rowave ablation treatment planning fusing radiomics and bioheat transfer modeling [11315-105]

1131533 Obtaining the potential number of object models/ atlases needed in medical image analysis [11315-106] 


\section{Authors}

Numbers in the index correspond to the last two digits of the seven-digit citation identifier (CID) a rticle numbering system used in Proceedings of SPIE. The first five digits reflect the volume number. Ba se 36 numbering is employed for the last two digits and indicates the order of articles within the volume. Numbers start with 00, 01, 02, 03, 04, 05, 06, 07, 08, 09, 0A, 0B...0Z, followed by 10-1Z, 20-2Z, etc.

Ajani, Bhavya, IW

Akbari, Hamed, 1S

Al Efishat, Mohammad A., 1Q

Ali, Sharib, 14

Allen, Da niel R., 02

Allen, Peter ., 1Q

Alsha ra, Yasmeen, 2W

Anari, Jason B., OA

Antunes, J acob T., 30

Appleton, Tom, $\mathrm{OE}$

Armand, Mehran, 1C

Aronson, J oshua, OP

Askan, Gokce, 1Q

Asselin, Mark, 0Q, 16, 19

Attiyeh, Marc A., 1Q

Babic, Drazenko, 1X

Ba inbridge, Daniel, 10, 28

Bakas, Spyridon, 1S

Balachandran, Vinod P., 1Q

Bardosi, Zoltan, IY

Barker, Kevin, 2K

Barr, Colton, 16

Barr, Keiran, 2G

Bascom, Rebecca, $1 U$

Basturk, Olca, 1Q

Bax, J effrey, 1B, 2K

Baxter, J ohn S. H., OM

Bednarz, Bryan, OY

Beerbaum, Philipp, $1 \mathrm{~K}$

Beltran, Chris, 2F

Benassi, Thomas, 0D, 20

Bender, Evan, OD

Bera, Kaustav, 30

Berg, J ona h, 2C

Beskin, $\mathrm{V}$., OH, 2T

Bettati, Patric, OW

Bezerra, Hiram, 13

Bhardwaj, Aditya, IW

Bhati, Mahendra T., OL

Bhuiyan, MD Fiaz Islam, 0Z

Bilello, Mic hel, 15

Bisleri, Gianluigi, 2Q

Bly, Randall A., 2 L

Boctor, Emad M., OC

Boone, Nora, 2E

Borgard, Heather, $1 \mathrm{E}$

Brady, J ustin T., 30

Burstrom, Gustav, $1 X$

Caffo, B., 10
Cahill, PatrickJ., OA

Cai, Yunliang, 29

Cakir, Ahmet, 2V

Capostagno, S., OB

Camahan, Patrick, 10, 28, 2E

Carson, Matthew D., $2 Z$

Carton, Franç ois-Xavier, OS

Chabanas, Matthieu, OS

Chakraborty, J aya sree, 1Q

Cha kravorti, Srijata, 07

Chalian, Majid, OW

Chaurasia, Akash, OD

Chen, Elvis C. S., 02, 06, 10, 28, 2E

Chen, Xia ojun, 31

Chen, Zhang, 23, 2R

Chou, Yuting, 1Q

Choueib, Saleh, 2A

Clarke, Collin, 02

Cleary, $\mathrm{K} ., \mathrm{OH}, 2 \mathrm{~T}$

Condino, Sara, 06

Connolly, Laura, 0Q, 19

Comer, Stephen M., 2F

Cotin, Stepha ne, IV

Dakkak, J essica, 20

D'Angelica, Mic hael I., 1Q

Dangi, Shusil, 2Y

Daniel, Bruce, OL

Davatzikos, Christos, 15

Davis, Brian J ., 15

Dawa nt, Benoit M., 07

Deaton, Nancy, $0 Z$

Deisher, Amanda J ., 11, 2F, 34

Deng, Shujie, 28

Desai, J a ydev P., $0 Z$

De Silva, T., 0G , 10

de With, Peter H. N., 1X, 21

Dhou, Salam, 2S

Ding, Alice K., $1 G$

Do, Richard K. G., $1 \mathrm{Q}$

Doerr, S. A., 1O, 2B, 2M, 2P

Doggen, Carine J. M., 25

Dormer, J a mes D., OW, 0Z, 23, 2R

Drebin, J effrey A., $1 Q$

Durtsc hi, Maxwell S., 29

Edstrom, Erik, $1 \mathrm{X}$

Egger, J an, 31

Ehtiati, T., OB

Eixmann, Tim, 04

Elang ovan, Sa njay, 20 
Elbata my, Lydia, 08

Ellerma nn, Katrin, 31

Elmi-Terander, Adrian, $1 X$

Engelhardt, Sa ndy, 0X, 1K

Emst, Floris, 04

Erus, Guray, 1S

Etkin, A mit, OL

Evans, Linton T., 29

Fan, Ca rolyn, OX

Fan, Xia oya o, 0O, OP, 29

Farshad, Mazda, 03

Fauver, Mark E., $2 Z$

Fei, Ba owei, OU, OW, 0Z, 2W

Fels, Sidney, 1E

Fenster, Aaron, 0E, 0K, 1B, 2I, 2K

Ferrari, Mauro, 06

Ferrari, Vincenzo, 06

Fiard, Gaelle, ol

Fic htinger, Gabor, 08, 0Q, 16, 17, 19, 24, 26, 27 ,

2A, 2G, 2Q

Figl, Michael, $1 Z$

Filippov, Mihail, 24

Foo, Thomas K., 0J , OY

Freysinger, Wolfgang, IY

Friebe, Micha el H., OT

Friedman, Kenneth, 30

Frisken, Sarah, 0J , IV

Fümstahl, Philipp, 03

Gaha n, J effrey, 2W

Gao, Cong, 1C

García-Vázquez, Verónic a, 04

Gardi, Lori, 1B, 2K

Geelkerken, Robert H., 25

Gharaibeh, Yazan, 13

Ghose, So umya, 0] , OY

Gillies, Derek J ., OE, OK, 2I, 2K

Ginty, Olivia, 2E

Golby, Alexandra, 0J , IV

Goldsmith, Louise, 14

Gonen, Mithat, $1 Q$

Gooding, J im, 09

Gormley, William B., 16

Grebenisan, Andrew, OR

Greil, Gerald, 1K

Grupp, Robert B., 1C

Gsaxner, Christina, 31

Gu, Xinyu, 2L

Gunacker, Simon, 31

Gupta, Soumya, 14

Gyac skov, lgor, 0K, $2 \mathrm{l}$

$\mathrm{Ha}$, Sung Min, $1 \mathrm{~S}$

Hager, G., 10

Halic ek, Martin, OU

Halter, Rya n J ., 1E, 1H, 2X

Han, R., OF, 1A, 10

Hannaford, Blake, 2L

Hanson, S. E., 2H

Haouchine, Nazim, IV

Hargreaves, Brian, OL

Ha rington, Kate A., 1 Q
Hasan, S. M. Kamrul, 1]

Hashtrudi-Zaad, Keyvan, 2Q

Hata, Nobuhiko, 2C

Haya shi, Yuic hiro, 18

Heiselman, J on S., 1F, 1G, 1L

Helm, P. A., 2B, 2M, 2P

Hendriks, Benno H. W., $1 X$

Herman, Micha el G., 11, 2F, 34

Herz, C hristian, 2E

Heslinga, Friso G., 25

Higgins, Willia m E., 1T, $1 U$

Hill, Matthew, OD

Hohma nn, S., 11, 34

Honma, Hirotoshi, 18

Hookey, Lawrence, 2G

Hossa in, Belayat, 1M

Hrinivich, W. Thomas, OK

Huang, Chuqin, $2 Z$

Huang, J a mes, OU, OW

Huang, Y., 2P

Hummel, J ohann, $1 Z$

Hummel, Marjan, 25

Hussa in, Ta rique, $1 \mathrm{~K}$

Hüttma nn, Gereon, 04

lommi, David, $1 Z$

Iwata, Hiroya su, 20

Izard, Jason, OR

J äckle, Sonja, 04

Jaeger, Mela nie, 2A

J amzad, Amoon, 0Q, 19

J annin, Pierre, OM

J a nssen, Natasja, 2A

J a rna gin, Willia rm R., $1 \mathrm{Q}$

Ji, Songbai, 29

J iang, Bote, 26

$\mathrm{J}$ in, Ze, 33

$J$ ithendra, Aditi, OD

J ohnson, Brett, 2W

J olley, Matthew A., 2E

J ones, C. K., 2B, 2M, 2P

J oschko, Abigayel, 02

J udson, Blake O., 2W

J upitz, Sydney, OY

J usomjai, Ka sidit, 32

J utras, J ean-David, 09

J uvekar, Pa rikshit, IV

Ka kani, Nimal, 2K

Ka minski, J a kub T., 1D

Kaufmann, Martin, $0 \mathrm{Q}$

Kekec, Tayg un, 12

Kenet, Adam, 20

Ketcha, M. D., OF, 1A, 1O, 2B, 2M, 2P

Khan, Mohammad M. R., 1R, 2U

King, Fra nklin, 2C

Kingham, T. Peter, $1 \mathrm{Q}$

Kita sa ka, Ta kayuki, 18

Kleemann, Markus, 04

Kleinszig, G., OF, 1A

Kloss, J onathan, OX

Kobashi, Syoji, 1M 
Koehler, Sven, 1K

Koffijberg, Hendrik, 25

Kokko, Mic hael A., $1 \mathrm{H}$

Kolen, Alexander F., 21

Kolluru, Chaitanya, 13

Konishi, H., 11, 34

Kronreif, Gemot, 26

Kruse, J on J ., 11, 2F, 34

Kuda velly, Srinivas Rao, 1W

Kuhlengel, Trevor K., $1 T$

Labadie, Robert F., 1R, $2 U$

Ladwa, Amrita, 20

La eseke, Pa ul F., 05, 15

La fra mboise, J acob, 17, 2G

Lai, Marco, $1 X$

Lapouge, Guillaume, 이

Lasso, Andras, 16, 19, 2E

Latus, Heiner, $1 \mathrm{~K}$

Lawrence, Sharon A., $1 \mathrm{Q}$

Le, Phuc, 2W

Lee, E. J ., $\mathrm{OH}$

Lee, J uhwan, 13

Lee, J ung hoon, Il

Lee, Lauren $\mathrm{K}$., $2 Z$

Lee, Sing Chun, 03

Leung, Lai Yee, 22

Leuze, C hristoph, OL

Li, Chen, OP, 29

L, Gen, 2R

Li, J ianning, 31

Li, Ming, OV

Li, Pan, 2T

L, Q inmei, 2W

L, Robert, OD

L, Xia ojian, 23, 2R

Ln, Sha n, $2 \mathrm{~L}$

Linte, Cristian A., 1] , $2 Y$

Lu, Simon, 20

Liu, Xinqi, 2C

Lu, Zteng, 2V

Loyd, J ohn, 1E

Long, Dilara, OV

Lott, Carina, OA

Luo, Ma, $1 \mathrm{~L}$

Mac Donald, Mic hael, oY

Maguet, Ehouam, OM

Maha devan, Eashwar, 20

Manba chi, Amir, OD, 20

Manni, Francesc a, 1X

Marinelli, Luca, 22

McCarren, Kathleen, OD

McDonough, J oseph M., OA

McGamy, Ciara, 2A

Mc Intyre, Ca itlin A., 1Q

McNab, J ennifer A., OL

Meerwaldt, Robbert, 25

Melito, Gian Marco, 31

Menard, Alexa ndre, OR

Merchant, F. A., 2H

Meszoely, Ingrid M., 1L
Miga, Micha el I., 1F, 1G, 1L

Mills, David, 0J , OY

Min, Lan, 21

Mirza, Sohail K., 29

Mistretta, Charles A., 15

Mitra, J himli, 0J , OY, 22

Mo, Lamy, 22

Moe, Kris S., 2L

Moelker, Adriaan, 12

Monfaredi, R., $\mathrm{OH}, 2 \mathrm{~T}$

Moore, J ohn, 02, 06, 10, 28, 2E

Mori, Kensaku, 18

Mori, Masaki, 18

Morrison, J onatha n, 22

Mousavi, Parvin, 09, 0Q, 0R, 19

Mukhopadhyay, So umik, IW

Müller-Stich, Beat Peter, OX

Munkvold, Bodil K. R., OS

Nam, Ha nna h H., 2E

Nanda, Siddhartha, 30

Na raya na n, Roshini, 20

Natori, Hiroshi, 18

Navab, Nassir, 03

Newman, L. K., 11, 34

$\mathrm{Ng}$, Kenneth, 20

Nickel, Felix, 0X

Nic ol, Christopher, 0Q, 19

Niessen, Wiro J ., 12

Nisar, Ha reem, 06

Nishio, Shoichi, 1M

Noble, J a ck H., 07, 0S, 1R, 2U, 2V

Nowroozilarki, Z, 2H

Oda, Masa hiro, 18

Oni, J ulius, OD

Orlando, Nathan, 21

Osgood, G. M., OF, OG, IA

Packer, D. L., 11, 34

Padala, Muralidhar, $0 Z$

Papemick, Sam, OE

Park, Claire K., 1B

Park, J un-Sung, IW

Parker, K. D., 11, 34

Paspulati, Raj M., 30

Pastel, David, 2X

Pathompatai, Chanok, 32

Patterson, Lindsey, 2A

Pätz, Torben, 04

Paulsen, Keith D., 0O, OP, 29

Paulussen, Elvira, 1X

Paydarfar, J oseph A., 1E, 2X

Pepe, Antonio, 31

Perdomo-Pa ntoja, A., 10

Periya samy, Sarvesh, 05, 15

Persson, Oscar, 1X

Peters, Temy M., 02, 06, 10, 28, $2 \mathrm{E}$

Pfefferle, Matthew, 2W

Piazza, Rob erta, 06

Pickardt, Thomas, $1 \mathrm{~K}$

Pieper, Steve, 16, 2T

Pigeau, Grace, 08, 27 
Pinter, Csaba, 26

Poignet, Philippe, 0 ol

Ponukumati, Aravind, $2 X$

Prabhu, David, 13

Prakash, Punit, 32

Preetha, C ha ndraka nth J ayac handran, OX

Pulvirenti, Alessandra, 1Q

Pushparajah, Kuberan, 28

Qiu, Catherine, OA

Quintanilla, Erick, 2X

Rafatza nd, Khashayar, 1D

Rahman, Nahian, $0 Z$

Rasmussen, Todd, 22

Rathore, Saima, 1S

Reece, G. P., 2H

Regodic, Milovan, $1 Y$

Reinertsen, Ingerid, OS

Rettmann, M. E., 11, 34

Richey, Winona L., $1 \mathrm{~L}$

Rittsc her, J ens, 14

Roberts, David W., 00

Robertson, Faith C., 16

Robinson, Adam, 1

Rodgers, J essica R., OK

Rozycki, Martin, 1S

Rubino, Rachel, 0Q, 19

Rudan, J ohn, 0Q

Sabah, Shafiya, 2S

Sa insbury, Ben, 24

Sako, C hiharu, $1 S$

Salomons, Gregory, 09

Sampathkumar, U., $2 \mathrm{H}$

Sánc hez, C. Antonio, 1E

Santilli, Alice, 26

Sarcar, Shourya, OY

Sarikouch, Samir, 1K

Sathya na raya na, Supriya, OL

Schonewille, Abigael, 08, 27

Schreiner, J ohn, 09

Sc hulz-Hildebrandt, Hinnerk, 04

Sebek, J an, 32

Sedghi, Alireza, 09, 0Q, 0R

Seibel, Eric J ., $2 Z$

Seibold, Matthias, 03

Seifabadi, Reza, oV

Seigne, J ohn D., $1 \mathrm{H}$

Shahbazi, Mahya, OC

Shahedi, Maysam, 0U, 0W, 2W

Shahub, Sarah, 2W

Shan, Caifeng, 1X, 21

Sharan, La lith, OX

Sharda, Sika nder, IW

Sharma, K., $\mathrm{OH}$

Sharma, Manuja, $2 Z$

Sheng, J un, $0 Z$

Sheth, N. M., OF, OG, IA

Shi, Yua n, 2X

Shinohara, Russell, $1 S$

Shukla, Gaurav, iS

$\mathrm{Si}$, Xiangyu, 2R
Siampli, E., 2T

Siddiq, Kamran, 20

Siemens, Robert, OR

Sieren, Malte Maria, 04

Siewerdsen, J. H., OB, OF, OG, 1A, 1O, 2B, 2M, 2P

Simpson, Amber L, 1Q

Simpson, J ohn M., 28

Singa na ma lli, Asha, 22

Singh, Ashish, 1S

Sisniega, A., OB, OG

Skolasky, R. L., 10

Skyman, Simon, 1X

Smith, L. Sc ott, 0J , OY, 22

Son, Yuri, IW

Song, Da niel Y., 11

Sotiras, Aristeid is, 15

Speidel, Michael A., 05, 15

Sramek, Michael, 2X

State, Claire, OD

Stayman, J. W., OB

Strother, Charles M., 15

Sultana, Sharmin, 1l

Sun, Changjian, OA

Sun, Yua nyua $\mathrm{n}, 12$

Sund erla nd, Kyle, 17, 24, 27

Swamy, Aka sh, 1X

Taeprasa rtsit, Pinyo, 32

Ta ka batake, Hirotsugu, 18

Takafunio, Hiranaka, 1M

Takasuka, Ha nna h, OD

Tand on, A nimesh, 1K

Taylor, Russell H., OC, $1 \mathrm{C}$

Tempany, Clare, OY

ter Mors, Thijs G., 25

Tessier, Da vid, 2K

Theodore, N., 10

Tian, Zhiqiang, 23, 2R

Tong, Yubing, OA

Torigian, Drew A., 0A, 33

Toth, J ennifer, $1 U$

Troccaz, J ocelyne, ol

Tryggestad, Erik J ., 2F

Tsumura, Ryosuke, 20

Tumey, Ben, 14

Udupa, J aya ram K., OA, 33

Unb erath, Mathias, $1 \mathrm{C}$

Uneri, A., OF, 0G, 1A, 1O, 2B, 2M, 2P

Ungi, Tamas, 08, 0Q, 17, 19, 24, 27, 2A, 2G, 2Q

Upendra, Roshan Reddy, 2Y

Vagdargi, P., OF, OG, IA

Valladares, Aleja ndra, $1 Z$

Van Citters, Douglas W., $1 \mathrm{H}$

van Walsum, Theo, 12

Vargas, J ose, 2W

Vedula, S. S., 10

Venug opal, Prem, 22

Vijayan, R. C., OF, 1A, 10

Viswanath, Satish E., 30

Vogt, S., OF, 1A

von Haxthausen, Felix, 04 
Wagner, Martin G., 05, 15

Wallace, Kirk, 22

Wan Chan Tseung, Hok Seum, 2F

Wang, Cheng, 18

Wang, J ianing, 07

Wehrtmann, Fabian Siegfried, OX

Weiss, C. R., OB

Wells, William M. III, 09, IV

Wetzstein, Gordon, OL

Wheeler, Gavin, 28

Wibowo, Henky, 32

Wiercigroch, J ulia, 2Q

Williams, Travis L, 1Q

Willis, J oseph E., 30

Wilson, David, 13

Witham, T., 10

Wolf, Ivo, $1 \mathrm{~K}$

Wood, Bradford J ., OV

Wu, Caiyun, OA

$\mathrm{Wu}, \mathrm{C}$ atherine $\mathrm{O}$., 24

Wu, P., OF, 1A, 2B

$\mathrm{Wu}$, Victoria, 08,27

Wu, Xia otian, 1E, 2X

Wu, Yixuan, $0 \mathrm{C}$

Xie, Tian, OC

$\mathrm{Xu}$, Sheng, OV

Yam, Sc ott, 0Q , 19

Yan, J emy, OD

Yan, J ustin, 20

Yang, Hongxu, 21

Yamolenko, P., $\mathrm{OH}$

Yates, Lauren, 19

Yeo, Desmond Teck-Beng, 0J , OY

Zevin, Boris, 17

Zhang, Haichong K., 1D

Zhang, $\mathrm{X}$, 2B, 2M, 2P

Zhang, Yinshu, 23

Zhang, Zheng, 2F

Zhao, Wennan, $1 \mathrm{U}$

Zhao, Yiyua $n, 07$

Zmin, Vladislav N., 13 
Proc. of SPIE Vol. 11315 1131501-16

Downloaded From: https://www.spiedigitallibrary.org/conference-proceedings-of-spie on 26 Apr 2023 Terms of Use: https://www.spiedigitallibrary.org/terms-of-use 


\title{
Conference Committee
}

\author{
Symposium Chairs
}

Georgia D. Tourassi, Oak Ridge National Laboratory (United States)

Metin N. Gurcan, Wa ke Forest Baptist Medical Center (United States)

Conference Chairs

Baowei Fei, The University of Texas at Dallas (United States) and The University of Texas Southwestem Medical Center (United States)

Cristian A. Linte, Roc hester Institute of Tec hnology (United States)

Conference Programme Committee

Purang Abolmaesumi, The University of British Columbia (Canada)

Matthieu Chabanas, Université Grenoble Alpes (France)

Evis C. S. Chen, Roba rts Resea rch Institute (Canada)

Sandrine de Ribaupieme, Westem University (Canada)

Gabor Fic htinger, Queen's University (Cana da)

Ryan J. Halter, Thayer Sc hool of Engineering at Dartmouth (United States)

David Hawkes, University College London (United Kingdom)

David R. Haynor, University of Wa shington (United States)

William E. Higgins, The Pennsylva nia State University (United States)

David R. Holmes III, Mayo Clinic (United States)

Pieme Jannin, Université de Rennes 1 (France)

David M. Kwartowitz, Grand Canyon University (United States)

Shuo Li, Westem University (Canada)

Michael I. Miga, Vanderbilt University (United States)

Kensaku Mori, Na goya University (J a pan)

Parvin Mousavi, Queen's University (Canada)

Jack H. Noble, Vanderbilt University (United States)

Maryam E. Rettmann, Mayo Clinic (United States)

Frank Sauer, Siemens Healthineers (United States)

Eric J. Seibel, University of Wa shington (United States)

Guy Shechter, Philips Hea Ithc are (United States)

Jeffrey H. Siewerdsen, J ohns Hopkins University (United States)

AmberL Simpson, Memorial Sloan-Kettering Cancer Center (United States)

Stefanie Speidel, Nationa le Centrum für Tumorerkrankungen Dresden (Gemany)

Tamas Ungi, La boratory for Perc uta neous Surgery (C a na da)

Satish E. Viswanath, Case Westem Reserve University (United States)

RobertJ. Webster III, Vanderbilt University (United States) 
Andrew D. Wiles, Northem Digital Inc . (Canada)

Ivo Wolf, Hoc hschule Mannheim (Gemany)

Zv R. Yaniv, National Institute of Allergy and InfectiousDiseases

(United States)

\section{Session Chairs}

1 Calibration and Tracking for Image-guided Navigation

Evis C. S. Chen, Robarts Research Institute (Canada)

Tamas Ungi, La boratory for Perc uta neous Surgery (Canada)

2 Al Methods for Image-guided Therapy

Jack H. Noble, Vanderbilt University (United States)

Matthieu Chabanas, Université Grenoble Alpes (France)

3 Image-guided Orthopedic Applications

David R. Haynor, University of Wa shington (United States)

David R. Holmes III, Mayo Clinic (United States)

4 Ultrasound Imaging and Image Guidance: J oint Session with

Conferences 11315 and 11319

Haichong Kai Zhang, Worcester Polytec hnic Institute (United States)

Parvin Mousavi, Queen's University (Canada)

5 Image-guided Neurosurgic al Interventions

Ivo Wolf, Hoc hschule Mannheim (Gemany)

David M. Kwartowitz, Grand Canyon University (United States)

7 Al-based Methods for Tissue Classific ation: Diagnosis and Therapy Applications

Jeffrey H. Siewerdsen, J ohns Hopkins University (United States)

8 Keynote Presentation

Cristian A. Linte, Rochester Institute of Tec hnology (United States)

Baowei Fei, The University of Texas at Dallas (United States)

9 Augmented Reality for Image-guided Therapy

Eric J. Seibel, University of Wa shington (United States)

Maryam E. Rettmann, Mayo Clinic (United States)

10 Novel Imaging Technologies for Interventional Guidance

David R. Holmes III, Ma yo Clinic (United States)

Zv R. Yaniv, National Institute of Allergy and Infectious Diseases

(United States) 
11 Video and Optical Methods for Imaging

William E. Higgins, The Pennsylva nia State University (United States)

Ryan J . Halter, Thayer Sc hool of Engineering at Dartmouth (United States)

12 Robot-assisted Image-guided Therapy

Zv R. Yaniv, National Institute of Allergy and Infectious Diseases

(United States)

Evis C. S. Chen, Robarts Research Institute (Canada)

13 Modeling Applic ations for Image-guided Thera peutics

Matthieu Chabanas, Université Grenoble Alpes (France)

Maryam E. Rettmann, Mayo Clinic (United States)

14 Al-based Image Segmentation and Feature Detection

Kensaku Mori, Nagoya University (J a pan)

Satish E. Viswanath, Case Westem Reserve University (United States)

15 J oumal of Medic al Imaging Special Section on Interventional Data Science

Amber L Simpson, Queen's University (United States)

Michael I. Miga, Vanderbilt University (United States) 
Proc. of SPIE Vol. 11315 1131501-20

Downloaded From: https://www.spiedigitallibrary.org/conference-proceedings-of-spie on 26 Apr 2023 Terms of Use: https://www.spiedigitallibrary.org/terms-of-use 


\section{Medical Imaging Award Recipients}

\section{Robert F. Wagner Best Student Paper Award}

Robert F. Wagner was an active scientist in the SPIE Medical Imaging meeting, starting with the first meeting in 1972 and continuing throughout his career. He ensured that the $\mathrm{BRH}$, and subsequently the $\mathrm{CDRH}$, was a sponsor for the early and subsequent Medical Imaging meetings, helping to launch and ensure the historical success of the meeting. The Robert F. Wagner All-Conference Best Student Paper Award (established 2014) is acknowledgment of his many important contributions to the Medical Imaging meeting and his many important advances to the field of medical imaging.

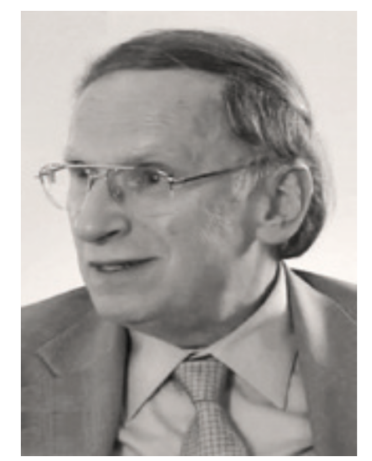

This award is co-sponsored by:

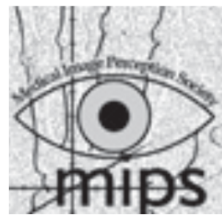

The Medical Image Perception Society

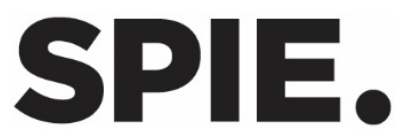

2020 Recipients:

First Place: Multi-body registration for fracture reduction in orthopaedic trauma surgery (11315-14)

R. Han, A. Uneri, P. Wu, R. Vijayan, P. Vagdargi, M. Ketcha, N. Sheth, Johns Hopkins University (United States), S. Vogt, G. Kleinszig, Siemens Healthineers (Germany) G. M. Osgood, John Hopkins Hospital (United States), J. H. Siewerdsen, John Hopkins University (United States)

Second Place: Phase contrast CT enabled three-material decomposition in spectral CT imaging (11312-47)

Xu Ji, Ran Zhang, Ke Li, Guang-Hong Chen, University of Wisconsin School of Medicine and Public Health (United States)

Image-Guided Procedures, Robotic Interventions, and Modeling Young Scientist Awards sponsored by Siemens Healthineers

Winner: Development of ultrasonography assistance robot for prenatal care (11315-90)

Ryosuke Tsumuraa, Waseda University (Japan) and Worcester Polytechnic Institute (United States), Hiroyasu Iwata, Waseda University (Japan)

\section{Runner-up: Motion induced segmentation of stone fragments in ureteroscopy video} (11315-39)

Soumya Gupta, Sharib Ali, University of Oxford (United Kingdom), Louise Goldsmith, Ben Turney, Oxford University Hospitals NHS Trust (United Kingdom), Jens Rittscher, University of Oxford

(United Kingdom) 
Proc. of SPIE Vol. 11315 1131501-22

Downloaded From: https://www.spiedigitallibrary.org/conference-proceedings-of-spie on 26 Apr 2023 Terms of Use: https://www.spiedigitallibrary.org/terms-of-use 